\title{
Virtual Computed Tomography Cystoscopy in Bladder Pathologies
}

\author{
Halil Arslan, Kadir Ceylan, Mustafa Harman, Yuksel Yilmaz, Osman Temizoz, Saban Can \\ Departments of Radiology and Urology, Yuzuncu Yil University School of Medicine, Van, Turkey
}

\begin{abstract}
Objective: Assessed the usefulness of virtual cystoscopy performed with multidetector computed tomography (CT) in patients with different urinary bladder pathologies compared to the conventional cystoscopy.

Materials and Methods: Eighteen patients with different bladder pathologies, which consisted of 11 tumors, 3 diverticula, 2 trabecular changes and 2 stones, were assessed with conventional cystoscopy and virtual CT cystoscopy. The results of virtual CT cystoscopy were compared with the findings of conventional cystoscopy. We determined the detection rate and positive predictive value of CT imaging based virtual cystoscopy in the diagnosis of urinary bladder lesions.

Results: CT scanning was well tolerated by all patients, and no complications occurred. Images in 16 (88\%) of the 18 virtual cystoscopic examinations were either of excellent or good quality. All tumors except one, 2 trabecular changes and 2 stones were characterized with similar findings in the both of methods. The masses ranged from 0.4 to $7.0 \mathrm{~cm}$ in diameter. While conventional cystoscopy could not evaluate interior part of the diverticulum, virtual CT cystoscopy could demonstrate clearly within it. There were no false-positive findings in our series.

Conclusion: Virtual CT cystoscopy is a promising technique to be used in the detection of bladder lesions. It should be considered especially at the evaluation of bladder diverticula. In the future, it may be possible or even advantageous to incorporate into the imaging algorithm for evaluation of bladder lesion.
\end{abstract}

Key words: bladder; cystoscopy; tomography, spiral computed; tumors; diverticula; stones Int Braz J Urol. 2006; 32: 147-54

\section{INTRODUCTION}

Bladder pathologies are consisted of the important group of genitourinary tract diseases. The most common complaints in bladder disease are microscopic and macroscopic hematuria, disuria and other voiding symptoms. All these symptoms may be related to inflammatory, neoplastic, stones, neurologic, obstructive or congenital abnormalities. Urogram, sonography (US), computed tomography (CT), magnetic resonant imaging (MRI) and some other radiological modality have been used for a long time in all these pathologies. However, conventional cystoscopy is a standard diagnostic approach for urinary bladder evaluation, its primary indication is the diagnosis of lower urinary tract disease, sings, and symptoms that may be related to the urinary tract are evaluated using cystoscopy to directly visualize lower urinary tract anatomy and macroscopic pathology. However, this procedure has drawbacks, including its high costs and an invasiveness that may lead to iatrogenic bladder injury and urinary sepsis. CT is usually recommended as a useful radiologic approach for assessing bladder disease, but CT has low sensitivity for detection of small bladder lesions. For CT to depict a small bladder lesion, optimal imaging conditions, including 
adequate bladder distention and thin-slice scanning, must be satisfied. Therefore, negative findings on CT warrant performance of conventional cystoscopy in patients with bladder pathology (1-4).

Recently, three-dimensional computer-rendering techniques with rapid image acquisition have led to the development of virtual-reality imaging. With commercially available software, virtual reality imaging allows interactive intraluminal navigation through any hollow viscus, simulating conventional cystoscopy. This technique of virtual endoscopy has been applied to many organs, including the colon, bronchus, stomach, and bladder (3-6).

Currently, most authors have been studied with virtual cystoscopy about the bladder tumor. Few reports are found in the literature regarding different bladder pathology such as diverticulum or inflammatory pseudotumor based virtual cystoscopy of the urinary bladder $(7,8)$.

The purpose of this study was to evaluate the usefulness of virtual cystoscopy using a volume rendering algorithm performed with multidetector CT in patients with different urinary bladder pathology compared with the gold standard that is, conventional cystoscopy and to determine the modality's detection rate and positive predictive value.

\section{MATERIALS AND METHODS}

Eighteen patients (mean age $56 \pm 11$ years, range 40 to 72 years) were referred from the urology department because of the different urinary bladder disease, which consisted of 11 tumors, 3 diverticula, 2 trabecular changes and 2 stones for this study. We carried out both conventional and virtual cystoscopy in all patients. Time interval between conventional and CT cystoscopy ranged from zero to 7 days. Each patient had various clinical histories. Most of the patients presented painless hematuria or dysuria. Conventional cystoscopies were carried out with unaware of virtual cystoscopic findings. The conventional cystoscopies were performed with rigid $21 \mathrm{~F}$ cystoscope (Storz, Germany) with a field of view of 30 degrees in all patients under general or local anesthesia. Virtual cystoscopic examinations were started with obtaining adequate bladder distention in supine posi- tion. Helical CT was performed with 4 channel CT scanner (Somatom Sensation 4, Siemens Medical Systems, Erlangen, Germany), in single breath hold, with $1 \mathrm{~mm}$ collimation, $1 \mathrm{~mm}$ reconstruction interval and $3 \mathrm{~mm}$ thickness. Other scanning parameters were as follows: $1 \mathrm{~mm}$ reconstruction interval, $\mathrm{mAs} 153$, and $120 \mathrm{kV}$, feed/rotation $5 \mathrm{~mm}$. The scanning time was only 8-12 second. Prior to the scan, adequate filling of the bladder with approximately $250-450 \mathrm{~mL}$ of air was required. At the same time, IV $100 \mathrm{~mL}$ contrast medium was administered in all patients by a power injector at a rate of $2.0-2.5 \mathrm{~mL} / \mathrm{s}$ for possible extravesical invasion of the tumor or some other pathology. The patients were then turned to the prone position, and CT of the bladder was repeated with use of the same parameters after a repeated scout view was obtained. Additional bladder distention with approximately $80-120 \mathrm{~mL}$ of air was necessary in some of the patients, since repositioning led to leakage of some of the insufflated gas from the bladder.

The data were downloaded to an independent workstation (Leonardo; Siemens Medical Systems) equipped with software for interactive intraluminal navigation. Using multiplanar reformation from source images, a central observation point was defined in the middle of the lumen of the bladder. The camera for virtual cystoscopy was placed in the center of the bladder lumen and thereafter was advanced to each quadrant in turn. When a possible abnormality was discovered, it was fully evaluated from various angles.

The number, location, and size of the tumors were individually determined and noted after cystoscopy for later comparison with the results of CT imaging-based virtual cystoscopy. The virtual and conventional cystoscopic findings for each patient were documented on separate worksheets. The number, size, location, and morphologic features of the masses, mucosal thickness, trabeculations and diverticula and other luminal pathology were also noted in both methods. On transverse section and virtual CT images obtained with the patients in both supine and prone positions, each mass lesion was characterized as a focal polypoid lesion, a sessile mass, or wall thickening. A discrete lesion was considered polypoid, if it was taller rather than wider, while a sessile mass was 
defined as a lesion when it was wider at the base. A lesion was characterized as wall thickening when there was elevation of the bladder wall without a discrete mass. The quality of each CT image was also evaluated in terms of the residual urine, which may obscure the bladder mucosa, and the degree of distention. Complications due to CT cystoscopy were recorded.

Three radiologists (HA,MH,OT) blinded to the findings of conventional cystoscopy, independently interpreted the images prospectively, and any discrepant readings were resolved by consensus. The results of virtual CT cystoscopy were compared with the findings of conventional cystoscopy, which is considered the standard. The lesions that were not prospectively identified at CT cystoscopy were retrospectively evaluated for visibility on transverse and virtual images. The pathology report in each patient with bladder tumor was also reviewed for further correlation. Using conventional cystoscopy as the gold standard, we analyzed them to determine the detection rate of CT imaging-based virtual cystoscopy in the diagnosis of urinary bladder lesions.

\section{RESULTS}

CT scanning was well tolerated by all patients, and no complication occurred. Images in 16 (88\%) of the 18 virtual cystoscopic examinations were of excellent or good quality, with adequate bladder distention and minimum residual urine. Images in 2 examinations were suboptimal due to either moder- ate residual urine or inadequate bladder distention. Tumoral lesions were seen in one of them. However, in other patient, a smaller-than-4 $\mathrm{mm}$ polypoid tumoral lesion could not be detected.

On conventional cystoscopy, 11 tumors were found in 18 patients. These masses ranged from 0.4 to $7.0 \mathrm{~cm}$ in diameter (mean, $1.5 \mathrm{~cm}$ ). Out of 11 polypoid lesions, 8 were larger than $5 \mathrm{~mm}$, and 3 were 5 $\mathrm{mm}$ or smaller. Eight out of the polypoid lesions were larger than $5 \mathrm{~mm}$, and 3 were $5 \mathrm{~mm}$ or smaller. One of the polypoid lesions was calcified. Six out of the 11 lesions were located on the lateral wall (Figures-1 and 2); 2 on the posterior wall; 2 on the anterior wall and 1 in the bladder neck. All lesions were diagnosed as transitional cell carcinoma in the pathology reports. All these tumors had been described by the virtual cistoscopy with nearly similar findings in size localization and surface of the tumor except one lesion, which was smaller than $5 \mathrm{~mm} 90 \%$ of the tumors were diagnosed by virtual cystoscopy as compared to conventional cystoscopy. Mucosal thickness and trabeculations were also seen in the virtual CT cystoscopy and the appearance was similar in both modalities (Figure-3). Bladder stone could not differentiate the tumor or polyp without adjustment of the lowest and highest point of the density value in the volume-rendering method in two patients (Figure-4).

Three diverticula were diagnosed, but the interior of the diverticula could not be evaluated by conventional cystoscopy. Their lumens were easily detected by virtual cystoscopy. In three diverticula, virtual CT cystoscopies were superior to conventional
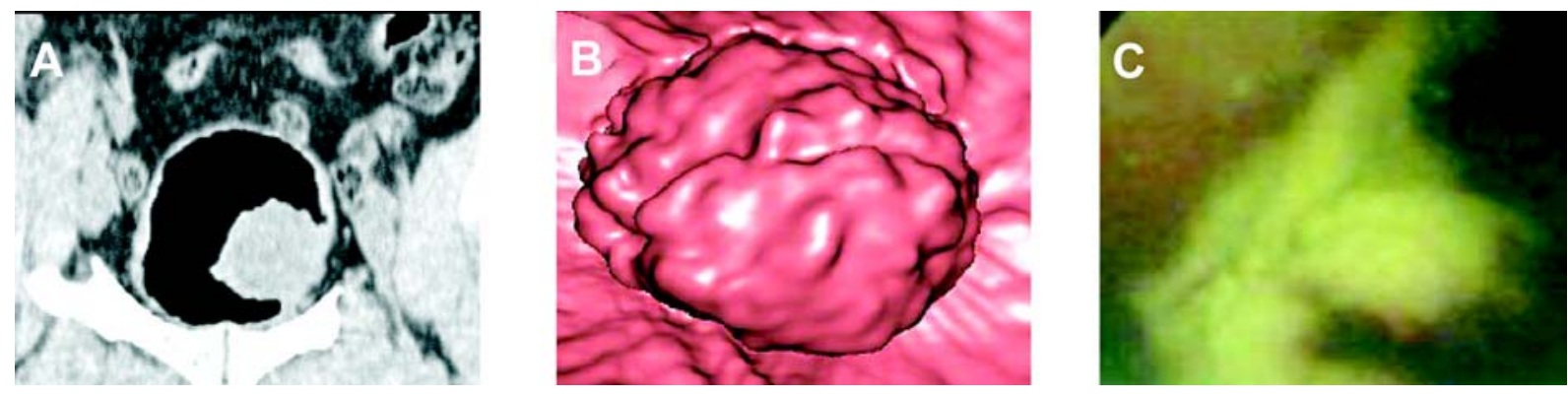

Figure 1 - 50-year-old man with transitional cell carcinoma obtained in area toward left wall shows polypoid lesion. A) Coronal multiplanar reconstruction section. B) Virtual CT cystoscopy appearance. C) Conventional cystoscopy. Vegetative surface of the tumor was clearly identified in virtual CT cystoscopy. Surrounding mucosal surface appears normal both of the conventional and virtual CT cystoscopy. 

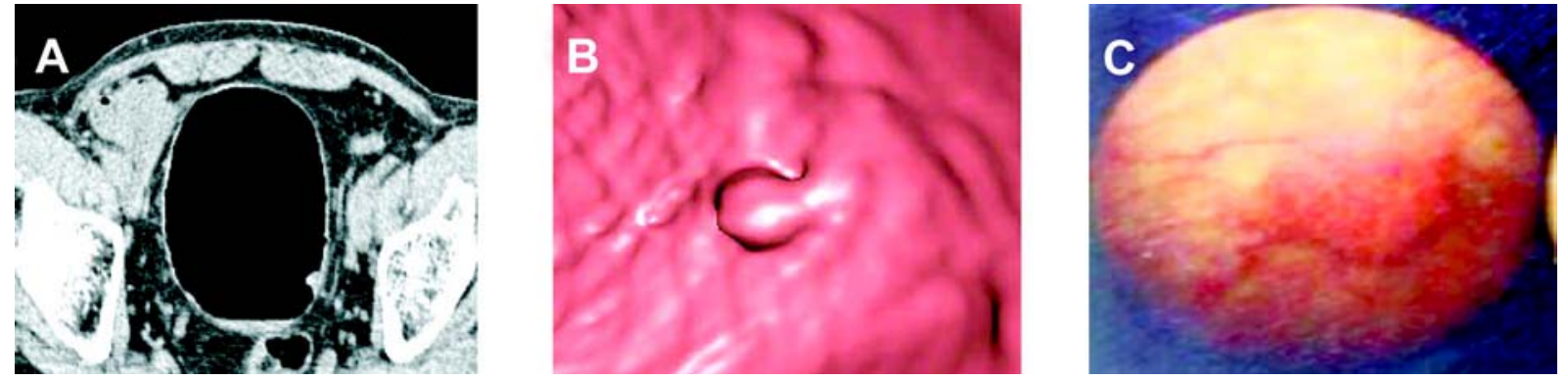

Figure 2 - 60 year-old man with primary urinary bladder cancer. A) Transverse section. B) Virtual CT cystoscopy appearance. C) Conventional cystoscopy. Virtual CT cystoscopic image focused on polyp with $12 \mathrm{~mm}$ located near left urethral orifice. Internal urethral orifice can be identified only polyp with $12 \mathrm{~mm}$ located near left urethral orifice. Internal urethral orifice can be identified in lower midportion.
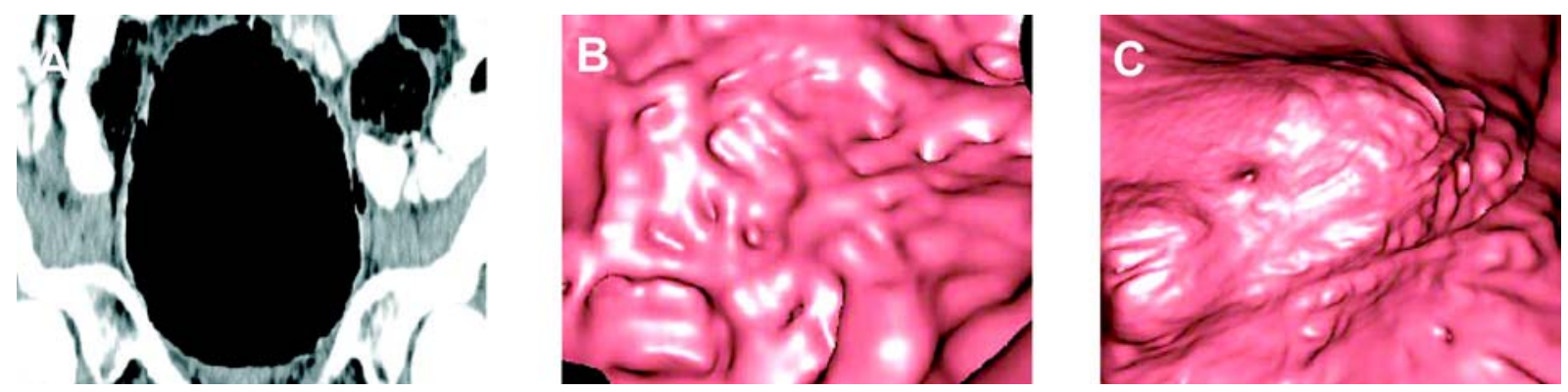

Figure 3 - 71 year-old man with trabeculation because of the prostate hypertrophy. A) Coronal multiplanar reconstruction image. B) Magnified virtual CT cystoscopy appearance. C) General trabecular appearance of mucosal surface in virtual CT cystoscopy. Virtual CT cystoscopy shows mucosal thickness and trabeculation similar with the conventional cystoscopy.
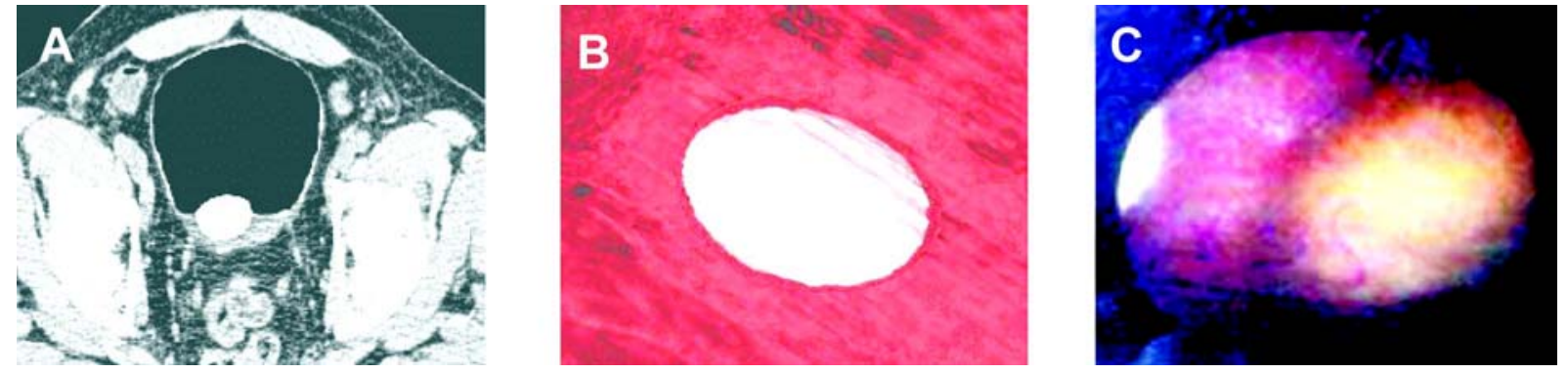

Figure 4- 55 year-old man with solitary bladder stone. A) Transverse section. B) Virtual CT cystoscopy appearance. C) Conventional cystoscopy. Stone could be easily differentiated from the surrounding tissue in virtual CT cystoscopy appearance after the adjustment threshold value of density.

cystoscopy in demonstration of the interior of the diverticula (Figure-5).

Transverse section and virtual CT images were complementary in lesion detection and characterization. Although areas of wall thickening and trabeculation were seen on the virtual images, they were more conspicuous on the transverse views. However, the lobulated morphologic characteristics of a small polypoid lesion were better depicted on the virtual image. There were no false-positive findings in our series. The presence of every lesion seen at virtual cystoscopy was confirmed at conventional cystoscopy. Virtual cystoscopy time, including catheter placement, was approximately 20-25 minutes. Con- 

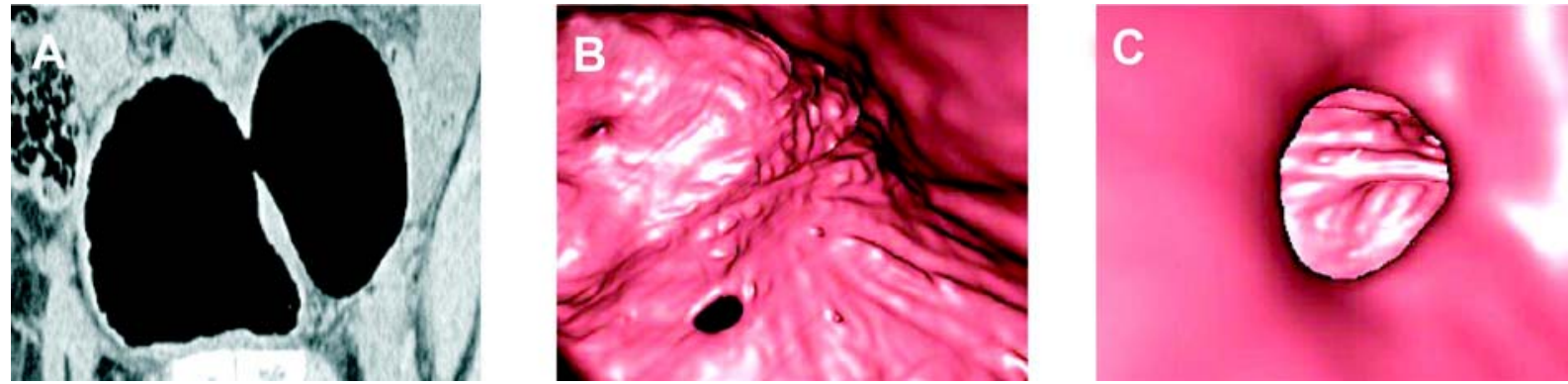

Figure 5-45 year-old men with virtual CT cystoscopic images of bladder wall diverticulum. A) Sagittal multiplanar reconstruction image. B) Virtual CT cystoscopy appearance of the neck of the diverticulum. C) Interior appearance of the diverticulum. Diverticulum could be evaluated both of the multiplanar reconstruction images and virtual CT cystoscopy, but could not in the conventional cystoscopy.

sidering conventional cystoscopy to be the gold standard, we found the following diagnostic values for the identification of bladder lesions on virtual cystoscopy. In 17 out of 18 patients (94.4\%), lesions were detected by using virtual CT cystoscopy.

\section{COMMENTS}

Several imaging techniques are available for use in the detection of bladder pathology. US, urogram, CT, MRI and conventional cystoscopy could be used in the bladder disease. Conventional cystoscopy was accepted as a gold standard in bladder $(3,5)$. However, there are several disadvantages of the conventional cystoscopy. It is often difficult to perform adequately when exploring the anterior bladder wall or a diverticulum cavity. Primary intradiverticular carcinomas are rare, but diagnosis is often difficult with conventional method $(6,9,10,11)$. There are some contraindications for the conventional cystoscopy such as bacteriuria, acute cystitis, urethritis, prostatitis, obstructive prostatic hypertrophy, and stricture or rupture of the urethra. Marked hematuria is another factor that limits the technical success of cystoscopy, thereby decreasing its reliability. On the other hand, cystoscopy is performed in general or local anesthesia and it is an invasive and uncomfortable procedure for patients, and complications such as infections, uretral or bladder perforation, scarring, and stricture of the urethra have been observed (3,6,12-14).

Virtual endoscopy is a recently developed noninvasive method to detect tumors protruding from the walls of hollow organs. A promising advantage of this imaging modality is that views not possible in conventional endoscopic examination can be created. The volumetric data obtained with helical CT or MR imaging are computer-rendered to generate three-dimensional images, and with commercially available software, intraluminal navigation through any hollow viscus is possible. There are two main techniques for the reconstruction of virtual image. One of them is volume rendering and the other is surface-rendering algorithm. Of the different three-dimensional rendering techniques available, the perspective volume rendering provides more information because the entire data set is incorporated (15-19). We used a volumerendering algorithm in this study.

Virtual endoscopy has been most widely applied to imaging of the colon and many investigators report its feasibility in the depiction of colorectal polyps $(20,21)$. After the first report of virtual cystoscopy, in the study by Vining at al., there have been a lot of studies on the utility of virtual cystoscopy of the bladder. The urinary bladder is a good candidate for virtual cystoscopy because of its simple luminal morphology, its relatively small volume, and the absence of involuntary peristalsis. Therefore, a virtual cystoscopic rendering of the bladder takes a short time to navigate and does not require great skill on the part of the operator $(4-6,18)$. On the other hand, according to a study by Kim et al., virtual cystoscopy was found superior than multiplanar reconstruction and source CT images for lesion detection in the contrast material-filled bladder (22). However, most stud- 
ies were performed in bladder tumor and previous studies have focused solely on known bladder lesion. There have been no enough studies on different pathologies. We carried out this study on different bladder pathologies and evaluated the capabilities of virtual cystoscopy, such as diverticula, trabeculation and stone.

As a minimally invasive procedure, virtual CT cystoscopy provides many advantages as compared to conventional cystoscopy. The virtual CT cystoscopy images could be stored in file and the lesion could be compared in follow up period with based images. The size of a tumor is measured objectively. Access to the anterior bladder wall or the lumen of a diverticulum is not restricted in virtual cystoscopy because various software reconstruction tools can be used and the tumor can be easily detected (23). Patients with a severe urethral stricture or marked prostatic hypertrophy, who may be poor candidates for conventional cystoscopy, can safely undergo virtual CT cystoscopy. It is also indicated for patients who are at risk of complications such as hemorrhage, perforation, infection, or pain, and for the examination of young patients $(3,5,6)$. In our group, diverticula were very well examined by virtual CT cystoscopy while conventional cystoscopy could not evaluate the interior of lesions. However, we had some difficulties in bladder stone using virtual cystoscopy. It was very difficult to differentiate the polyp without setting the threshold density value. This also showed us that axial images and virtual cystoscopy images should be evaluated together.

Two techniques have been used to obtain the CT source data for reconstructed virtual cystoscopic images, scanning the bladder that has been filled with either air or contrast material. Both methods have some advantages and disadvantages when compared with one another. Most previous studies have been chosen to scan the air-filled bladder. However, virtual cystoscopy of the air-filled bladder is inherently invasive because catheterization is required to introduce air into the bladder. Supine and prone examination is another disadvantage of the air-filled bladder method. On the other hand, filling the bladder with IV contrast material has been easily achieved in many studies. In this method, there is no need for examination in prone and supine position. Therefore, this means lesser radiation and cost $(3,22)$. However, urine and contrast could not be mixed properly with this method for virtual cystoscopy. This is one of the disadvantages. Secondly, IV contrast application is mandatory and this is another difficulty. Waiting for bladder filling and inadequate distention is another disadvantage. In addition to these disadvantages, possible scheduling problems may arise in a busy CT practice because of the repeated patient positioning and scanning required $(3,4,5)$. In our study, like many others, we used air-filled bladder for virtual cystoscopy. If there is already a Foley catheter inside the bladder, the air-filled bladder method might be preferable. However, when there is no catheter and a IV contrasted examination has already been planned, the second method can be used for virtual cystoscopy.

Virtual cystoscopy has several limitations. A major limitation is that it is unable to depict flat lesions, which appear as subtle mucosal color changes on conventional cystoscopy. However, various factors influence the detection of sessile lesions, including the method used to acquire the CT data, interactive navigational skill of the operator, attenuationcoefficient ranges used for voxel categorization, and degree of bladder distention. Sessile lesions usually have an irregular surface that must be minutely depicted on virtual cystoscopy. So if the examination parameter is appropriate and distention is adequate, sessile lesions also could be easily detected. Secondly, the differentiation between small tumors and inflammatory swelling of the mucosa could be difficult, especially in patients with unsatisfactory bladder filling. Inflammatory swelling of the mucosa thus could be misdiagnosed as a tumor, or small tumors could be missed on virtual cystoscopy. Insufficient distention of the bladder may also cause the mucosa to wrinkle. Third, mucosal thickening secondary to fibrosis cannot be distinguished from a neoplasm. Of course, one faces a similar problem on conventional cystoscopy because biopsy is often required to determine whether a bladder lesion is inflammatory, fibrotic, or neoplastic. A fourth disadvantage of virtual cystoscopy is that it lacks the ability to provide tissue for histologic evaluation, an ability that is possible on conventional cystoscopy and biopsy. Fifth, it is difficult to visualize the lumen of the urethra as is routinely done with conventional cystoscopy. How- 
ever, it was reported that urethra could also be evaluated by virtual cystoscopy (24).

In conclusion, virtual CT cystoscopy is a promising technique for tumor and some other bladder lesions, such as diverticula. Virtual CT cystoscopy is likely superior to demonstrate the interior part of the diverticulum. Adequate bladder distention and analysis of virtual images are required for optimal evaluation. This minimally invasive method can be of value for screening, primary diagnosis and surveillance of bladder lesions. Virtual CT cystoscopy may be indicated as a clinical routine when conventional cystoscopy is contraindicated or restricted in feasibility and interpretation or there is risk of hemorrhage, perforation, or pain especially in young patients. In the future, it may be possible or even advantageous to incorporate into the imaging algorithm for evaluation of bladder lesion through continued development and advancement of hardware and software. To determine the clinical value of virtual CT cystoscopy in the different bladder pathology, however, larger prospective studies in the general patient population are necessary.

\section{REFERENCES}

1. Carter HB: Basic Instrumentation and Cystoscopy. Walsh PC, Retik BA, Vaughan ED, Wein AJ (eds.). Campbell's Urology, Saunders, Philadelphia. 8 ed., 2002; vol. 1, pp. 111-21.

2. Bernhardt TM, Schmidl H, Philipp C, Allhoff EP, Rapp-Bernhardt U: Diagnostic potential of virtual cystoscopy of the bladder: MRI vs CT. Preliminary report. Eur Radiol. 2003; 13: 305-12.

3. Kim JK, Ahn JH, Park T, Ahn HJ, Kim CS, Cho KS: Virtual cystoscopy of the contrast material-filled bladder in patients with gross hematuria. AJR Am J Roentgenol. 2002; 179: 763-8.

4. Vining DJ, Zagoria RJ, Liu K, Stelts D: CT cystoscopy: an innovation in bladder imaging. AJR Am J Roentgenol. 1996; 166: 409-10.

5. Song JH, Francis IR, Platt JF, Cohan RH, Mohsin J, Kielb SJ, et al.: Bladder tumor detection at virtual cystoscopy. Radiology. 2001; 218: 95-100.

6. Lammle M, Beer A, Settles M, Hannig C, Schwaibold $\mathrm{H}$, Drews C: Reliability of MR imaging-based virtual cystoscopy in the diagnosis of cancer of the urinary bladder. AJR Am J Roentgenol. 2002; 178: 1483-8.
7. Zantl N, Beer A, van Randenborgh H, Hartung R: Virtual endoscopy of the urinary tract. Urologe A. 2002; 41: 552-8.

8. Sakamoto Y, Tanaka H, Kawabata G: Inflammatory pseudotumor of the urinary bladder diagnosed using 3DCT cystoscopy. Hinyokika Kiyo. 2003; 49: 587-90.

9. Durfee SM, Schwartz LH, Panicek DM, Russo P: MR imaging of carcinoma within urinary bladder diverticulum. Clin Imaging. 1997; 21: 290-2.

10. Stephenson WT, Holmes FF, Noble MJ, Gerald KB: Analysis of bladder carcinoma by subsite. Cystoscopic location may have prognostic value. Cancer. 1990; 66: 1630-5.

11. Baniel J, Vishna T: Primary transitional cell carcinoma in vesical diverticula. Urology. 1997; 50: 697-9.

12. Bavetta S, Olsha O, Fenely J: Spreading sepsis by cystoscopy. Postgrad Med J. 1990; 66: 734-5.

13. Mosbah A, Kane A, Zhani R, Hattab C: Iatrogenic urethral strictures of the male urethra. Acta Urol Belg. 1990; 58: 87-93.

14. Golomb J, Waizbard E, Iellin A, Merimsky E: Recurrent bladder perforation in chronic irradiation cystitis. J Urol. 1986; 92: 47-8.

15. Blezek DJ, Robb RA: Evaluating virtual endoscopy for clinical use. J Digit Imaging. 1997; 10(Suppl 1): 51-5.

16. Rubin GD, Beaulieu CF, Argiro V, Ringl H, Norbash AM, Feller JF, et al.: Perspective volume rendering of CT and MR images: applications for endoscopic imaging. Radiology. 1996; 199: 321-30.

17. Calhoun PS, Kuszyk BS, Heath DG, Carley JC, Fishman EK: Three-dimensional volume rendering of spiral CT data: theory and method. Radiographics. 1999; 19: 745-64.

18. Yazgan C, Fitoz S, Atasoy C, Turkolmez K, Yagci C, Akyar S: Virtual cystoscopy in the evaluation of bladder tumors. Clin Imaging. 2004; 28: 138-42.

19. Hopper KD, Iyriboz AT, Wise SW, Neuman JD, Mauger DT, Kasales CJ: Mucosal detail at CT virtual reality: surface versus volume rendering. Radiology. 2000; 214: 517-22.

20. Hara AK, Johnson CD, Reed JE, Ahlquist DA, Nelson H, MacCarty RL, et al.: Detection of colorectal polyps with CT colography: initial assessment of sensitivity and specificity. Radiology. 1997; 205: 59-65.

21. Chen SC, Lu DS, Hecht JR, Kadell BM: CT colonography: value of scanning in both the supine and prone positions. AJR Am J Roentgenol. 1999; 172: 5959.

22. Kim JK, Park SY, Kim HS, Kim SH, Cho KS: Comparison of virtual cystoscopy, multiplanar reformation, 
and source CT images with contrast material-filled bladder for detecting lesions. AJR Am J Roentgenol. 2005; 185: 689-96.

23. Prando A: CT-virtual endoscopy of the urinary tract. Int Braz J Urol. 2002; 28: 317-22.
24. Chou CP, Huang JS, Wu MT, Pan HB, Huang FD, Yu $\mathrm{CC}$, et al.: $\mathrm{CT}$ voiding urethrography and virtual urethroscopy: preliminary study with 16-MDCT. AJR Am J Roentgenol. 2005; 184: 1882-8.
Accepted after revision: January 25, 2006

\author{
Correspondence address: \\ Dr. Halil Arslan \\ YYÜ Týp Fakültesi Hastanesi \\ Radyoloji Anabilim Dalý \\ Maras. Cad, 65200, Van, TURKEY \\ Fax: + 90 432-2167519 \\ E-mail: drhalilarslan@ @otmail.com
}

\section{EDITORIAL COMMENT}

CT-cystoscopy has been shown to be a very accurate technique since it is able to detect lesions larger than $0.5 \mathrm{~cm}$ and is able to show mucosal abnormalities as small as $2 \mathrm{~mm}$.

CT-cystoscopy can be obtained either with gas-filled bladder or with contrast-material-filled bladder. Usually the sensitivity of this technique is higher for the detection of polypoid lesions in comparison with sessile lesions. In our institution we routinely evaluate the axial images together with virtual images since this combination, allows a significant increase in the overall sensitivity of this technique.

Virtual cystoscopy can also be obtained using magnetic resonance imaging (MR-cystoscopy). MR-cystoscopy has some advantages over CT-cystoscopy since there is no need for bladder catheterization or intravenous injection of contrast material, but has lower spatial resolution (better for lesions larger than $1 \mathrm{~cm}$ in diameter).

In this report, 18 patients with bladder pathologies were evaluated by CT-cystoscopy using distension of the bladder with gas after bladder catheterization. All patients were evaluated in both prone and supine position. The size of detected lesions ranged from 0.4 to $7.0 \mathrm{~cm}$ in diameter and there were no false-positive findings.

Bladder tumors can be noninvasively diagnosed using CT-cystoscopy or MR-cystoscopy, since both give comparable views to conventional cystoscopy. Virtual cystoscopy is helpful in cases where conventional cystoscopy is inconclusive or cannot be performed. One of the strengths of this technique is to add diagnostic information to conventional cystoscopy in the evaluation of bladder diverticula. Tumor within bladder diverticulum with narrow lumen can be easily demonstrated by virtual endoscopy.

Our goal for the future is to improve spatial resolution of CT-cystoscopy, reduce the radiation dose to the patient and provide useful information in order to allow conventional cystoscopy guided by the ctcystoscopic findings.

Dr. Adilson Prando Department of Radiology Vera Cruz Hospital Campinas, Sao Paulo, Brazil 